September 2014 Volume 2, Issue 1

(c) All rights are reserved by Derrick C Wan

\section{Ehlers-Danlos Syndrome and Skin Grafting}

\section{Introduction}

Ehlers-Danlos syndrome is a heritable connective tissue disorder with an estimated prevalence of one in 10,000 to 25,000 persons [1]. Using clinical phenotype, biochemical and molecular defects, as well as inheritance patterns, a total of 6 subtypes have been described [2]. Although its hallmark findings include severe joint laxity and hyperextensible skin, this condition is known to affect a variety of different organ systems, including the cardiovascular, gastrointestinal, and bronchopulmonary systems [3]. The underlying disorder is related to alterations of collagen type I, III, and V resulting in architectural changes and decreased cross-linking of collagen fibers [4]. This translates into decreased breaking strength of collagen with its clinical correlates being skin fragility, easy bruising, and susceptibility to minor trauma [3]. The concern for surgeons is primarily related to bleeding and poor wound healing, as patients with Ehlers-Danlos syndrome tend to develop wound dehiscence and delayed wound healing postoperatively $[5,6]$.

\section{Case Report}

Here we present a case of a 31-year old female patient with type 3 Ehlers-Danlos syndrome who presented to our institution with necrotizing soft tissue infection involving her right foot (dorsum) and lower leg. She was initially evaluated and treated by the general surgery service. Following repeated debridement, course of antibiotics including Bactrim and Rifadin as an outpatient, and negative pressure wound therapy, the patient was referred to the plastic surgery service for reconstruction of a $32 \times 27 \mathrm{~cm}$ wound involving her right lower leg and foot (Figure 1). The wound bed appeared adequately perfused. As such, the decision was made to pursue reconstruction by means of split-thickness skin grafting of the defect. A 0.012" split-thickness skin graft was harvested from the thigh. Of note, skin hyperextensibility made it quite challenging to harvest a confluent sheet of skin. After successful harvest of sheet grafts, $10 \mathrm{ml}$ of fibrin sealant (Tisseel, Baxter, Deerfield, IL) was applied to the wound bed, followed by inset of the skin grafts with 4-0 chromic sutures. A negative-pressure (75 $\mathrm{mmHg}$ continuous) wound dressing and an occlusive dressing (Tegaderm $^{\mathrm{TM}}$, 3M, St. Paul, MN) was applied to the skin graft and the donor site, respectively. The patient tolerated the procedure well. The postoperative course was rather unremarkable. After removal of the negative-pressure wound dressing on postoperative day 5, complete graft survival was noted. The patient was subsequently discharged home. At the first clinic appointment (2 weeks postoperatively), stable coverage of the initial defect was noted, with merely 2 small blisters seen under the skin graft. These were aspirated and the patient went on to heal her donor- and recipient sites uneventfully. At 1 month postoperatively, complete healing of all wounds was appreciated with hyperpigmentation of the donor- and recipient sites being the most notable clinical finding (Figure 2).

\section{Journal of}

Clinical \& Medical Case Reports

\section{Arash Momeni, and Derrick C Wan*}

Division of Plastic and Reconstructive Surgery, Stanford University Medical Center, USA

\section{Address for Correspondence}

Derrick C. Wan, Division of Plastic and Reconstructive Surgery, Stanford University Medical Center, 257 Campus Drive, Stanford, CA 94305, USA, E-Mail: dwan@stanford.edu

Submission: 05 August 2014

Accepted: 09 September 2014

Published: 15 September 2014

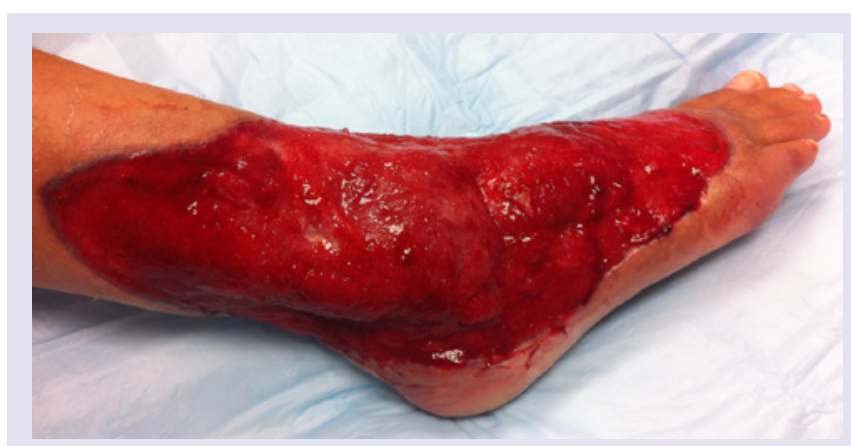

Figure 1: Photograph of right lower leg and foot wound following debridement and treatment with negative pressure wound therapy.
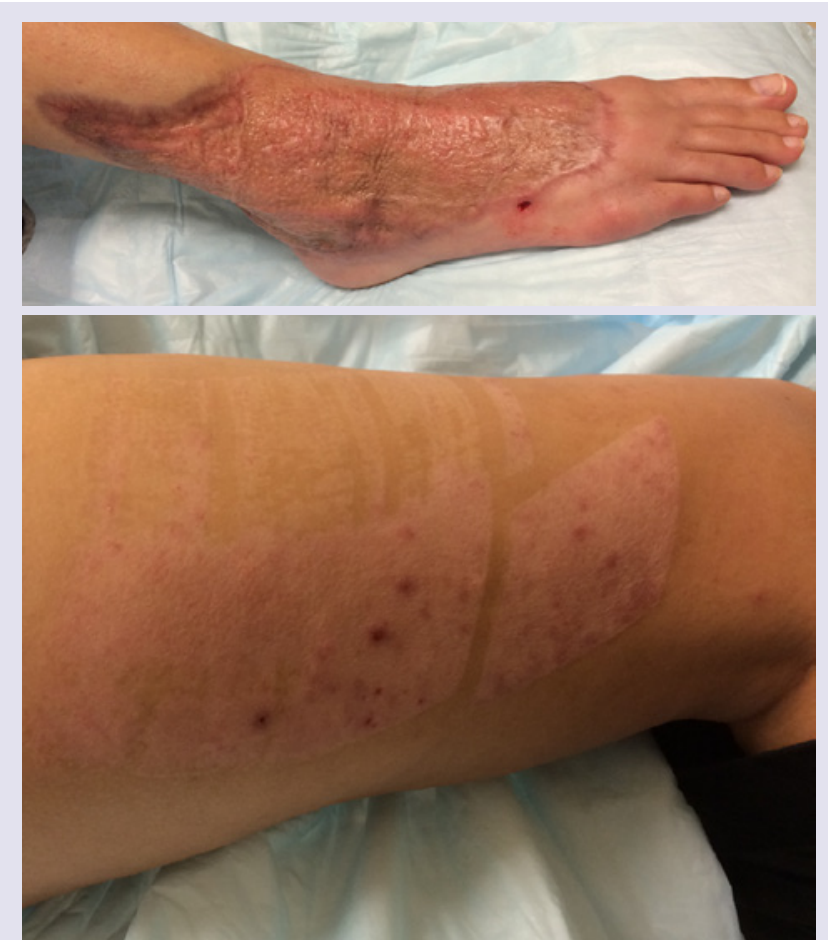

Figure 2: Photograph of right lower leg and foot wound (top) and right thigh split thickness donor site (bottom) at one month following skin grafting. 


\section{Discussion}

The main clinical features of patients with Ehlers-Danlos syndrome include skin hyperextensibility as well as skin fragility. While the former distinguishes Ehlers-Danlos syndrome from cutis laxa, the clinical manifestation of skin fragility is the propensity to develop skin tears that develop into atrophic scars [3]. The generalized weakness of the connective tissues is furthermore responsible for the characteristic joint hypermobility, which over time can be quite debilitating and difficult to manage, as well as the tendency to easily bruise. Given these features, surgical intervention in patients with Ehlers-Danlos syndrome should be approached with caution. In fact, Maltz et al suggest that "all surgical intervention should be as conservative as possible" with atraumatic tissue handling being mandatory [7].

Although numerous reports have been published discussing surgical treatment of patients with Ehlers-Danlos syndrome [5,7-9], only rarely has successful skin grafting been reported in patients with this condition $[4,10,11]$. The first case of successful skin grafting in the setting of Ehlers-Danlos syndrome was reported by Ricketson in 1957. Complete skin graft takes and uneventful wound healing of the donor site was noted [10]. Borris and colleagues reported a successful case of mandibular vestibuloplasty in a patient with Ehlers-Danlos syndrome [4]. While the recipient site developed suture-associated granulomas that healed after suture removal, the donor site displayed delayed wound healing, requiring a total of 6 months for complete healing to occur [4]. The most recent case of skin grafting in EhlersDanlos syndrome was reported by Kumar et al. [11]. A 15 x $10 \mathrm{~cm}$ right leg wound was treated successfully in a 22 -year-old male patient. Interestingly, similar to our case, the authors reported skin graft harvest to be challenging due to the hyperelastic properties of the skin. However, in their report, a limited access dressing (combination of intermittent negative pressure up to $30 \mathrm{mmHg}$ and a moist wound dressing) was instituted both for wound bed preparation and following skin grafting [11,12].

Surgical management of patients with Ehlers-Danlos syndrome is challenging due to alterations of collagen architecture and strength.
While our patient had type 3 Ehlers-Danlos which is typically associated with less severe skin manifestations, concern was still raised regarding her ability to generate an adequate granulation bed which would support an overlying skin graft. The present case, however, provides further evidence, that successful skin grafting is indeed possible and should be offered to patients who present with skin defects in the setting of a clean and well-perfused wound bed.

\section{References}

1. Buettner D, Hutchinson Fortier S (2009) Ehlers-danlos syndrome in trauma: A case review. J Emerg Nurs 35: 169-170.

2. Beighton P, De Paepe A, Steinmann B, Tsipouras P, Wenstrup RJ (1998) Ehlers-danlos syndromes: Revised nosology, villefranche, 1997. Ehlersdanlos national foundation (USA) and ehlers-danlos support group (uk). Am J Med Genet 77: 31-37.

3. Malfait F, De Paepe A (2014) The ehlers-danlos syndrome. Adv Exp Med Biol 802: 129-143.

4. Borris TJ, Bandrowsky T, Vorono AA (1997) Vestibuloplasty with a split thickness skin graft in a patient with ehlers-danlos syndrome. J Oral Maxillofac Surg 55: 419-423.

5. Ryan N, Walkden G, Akbar S (2012) Some wounds are hard to heal: An interesting presentation of ehlers-danlos syndrome. J Wound Care 223-226.

6. de Weerd L, Kjaeve J, Gurgia L, Weum S (2012) A large abdominal intercostal hernia in a patient with vascular type ehlers-danlos syndrome: A surgical challenge. Hernia 16: 117-120.

7. Maltz SB, Fantus RJ, Mellett MM, Kirby JP (2001) Surgical complications of ehlers-danlos syndrome type iv: Case report and review of the literature. J Trauma 51: 387-390.

8. Aldridge JM 3rd, Perry JJ, Osbahr DC, Speer KP (2003) Thermal capsulorraphy of bilateral glenohumeral joints in a pediatric patient with ehlers-danlos syndrome. Arthroscopy 19: E41.

9. Duthie G, Singh M, Jester I (2012) Laparoscopic management of colonic complications in ehlers-danlos syndrome type iv. J Pediatr Surg 47: e1-3.

10. Ricketson G (1957) The behavior of skin grafts and donor sites in a case of ehlers-danlos syndrome. Plast Reconstr Surg 20: 32-37.

11. Kumar P, Sethi N, Friji MT, Poornima S (2010) Wound healing and skin grafting in ehlers-danlos syndrome. Plast Reconstr Surg 126: 214e-215e.

12. Kumar P (2008) Limited access dressing. Wounds 20: 49-59. 